\title{
Containment Control of Heterogeneous Discrete-Time Multiagent Systems with Time Delay
}

\author{
Jiawei Wu $(\mathbb{D}$, Yongguang Yu $(\mathbb{D}$, and Guojian Ren \\ Department of Mathematics, Beijing Jiaotong University, Beijing 100044, China \\ Correspondence should be addressed to Guojian Ren; gjren@bjtu.edu.cn
}

Received 7 May 2021; Accepted 23 August 2021; Published 30 September 2021

Academic Editor: Rongwei Guo

Copyright (c) 2021 Jiawei Wu et al. This is an open access article distributed under the Creative Commons Attribution License, which permits unrestricted use, distribution, and reproduction in any medium, provided the original work is properly cited.

In this paper, the containment control of heterogeneous MASs with multi-interactional leaders is addressed. The objective of the containment control is of two layers. The leaders converge to an expected form; subsequently, the followers enter the convex hull spanned by the leader's final position. To achieve the goal, the dynamics of the leaders and the followers are modeled by a single integrator and a double integrator, respectively. A reduced-order transformation is employed to obtain the sufficient conditions for realizing the follower agents' control. In this manner, the maximum allowed time delay is given. Moreover, based on the topological structure and matrix, it confirms that the followers are able to enter the expected convex hull. Finally, the numerical simulation reveals the effectiveness of the control strategy.

\section{Introduction}

Over the past years, inspired by the work in [1-3], a lot of researchers began the research on multiagent systems (MASs) with cooperative control. The reasons lie on its widespread application in different disciplines [4-6]. In those works, the cooperative control in networks for MAS has been studied in the group behaviors. As one of the cooperative control, the containment control law, inspired from numerous natural and social phenomena, has attracted lots of researchers to continue developing it with the agents' neighbor information. Considering that the double-order controller dynamics has a good interpretation of the complex processes in the reality, its consensus control is of great interest, thus has been deeply studied [7-9]. More recently, the stabilization and consensus problem of systems has received much attention [10-12].

According to the number of leaders in the formation, the algorithm for its cooperative control can be demarcated into two categories: the case of one leader and the one with multiple leaders. As for the previous case, it is a consensus control problem. The corresponding algorithm will track the trajectory of each agents. Among these agents, one of them is called a leader, However, it is not able to receive any feedback from the other agents. There already exists many studies in the literature. In [13], the fixed-time leader-following consensus of second-order MASs with delay is investigated. In [14], a high-dimensional leader for neuroadaptive consensus tracking of MASs is discussed. In [15], a dynamic leader for bipartite tracking consensus of linear MASs is studied.

As for the case with several leaders, it focuses on the formation containing a group of leaders and followers. It is essentially a containment control problem. Different from the main idea in the consensus control problem, the control law in the containment control problem is supposed to force the followers to get into the convex hull formed by the leaders. In recent years, the containment control has received great population [16-18]. In [16], a dynamic output approach is investigated to study the containment of higherorder multileaders' MASs. In [17], a distributed containment law is proposed to study the double-integrator controllers for the multiple dynamic leaders by only using their position measurement. In [18], a distributed finite-time containment control is developed for the double-integrator MASs.

Many researchers have studied a group of leaders and followers. In recent years, the containment control problems [16-18] have been intensively studied, aiming at designing 
control laws to force followers into the convex hull formed by leaders. In [16], a dynamic output approach is investigated to study containment of higher-order multileaders MASs. In [17], distributed containment law is investigated to study multiple dynamic leaders for double-integrator dynamics and only using position measurement. In [18], a distributed finite-time containment control is investigated for double-integrator MASs.

However, the abovementioned algorithms only apply to the consensus problems with homogeneous MASs, in which the dynamics for all agents are modeled by the same order. Meanwhile, it should be mentioned that variety of phenomena for consensus problem cannot be described by the homogeneous dynamics systems [7, 19]. Indeed, the containment control problem of heterogeneous MASs has already been investigated in [20-22]. In [20], the asynchronous group consensus is investigated for heterogeneous MASs. In [21], a containment law is studied for heterogeneous MASs. In [22], a distributed containment law is studied for heterogeneous MASs.

Nevertheless, the protocols devised in the abovementioned references do not consider time-vary delay. Quan et al. and Dong et al. $[23,24]$ investigated MASs with time delay, but they are not for heterogeneous MASs. So, inspired by the above progress, the containment control problem of heterogeneous discrete-time MASs with time delay is considered in this manuscript. To be more specific, the leaders designed to form the formation are interactional. That is to say, the positions of leaders form the first layer of the MASs. Followers are controlled to enter the convex hull of the leaders' final position, in which the leaders are first-order agents and the followers are second-order agents. In view of the different goals of the leaders and the followers, this paper designs a leader position formation control protocol which is independent of the positions of the followers. Moreover, a consensus method based on the agents' neighbor information is proposed. The upper bound of time delay is given which depends on the topology, the control parameters, and the sampling time.

The paper is organized as follows. In Section 2, some important definitions on topology are given. In Section 3, the dynamics equation and control law are described and some basic lemmas are given. In Section 4, the stability of containment control law involving multiple leaders is studied. In Sections 5 and 6, the numerical simulation and conclusions are presented, respectively.

\section{Preliminaries}

In this section, some basic knowledge about graph, concerned definition, and notations is given.

2.1. Graph Theory. In the study of MASs, the directed graph is always employed for modeling the communication between agents. The graph offers an intuitive glance of the switching topology, and it also facilitates the control analysis. Basically, the network consists of $n$ agents ( $m$ leaders and $n-m$ leaders), and its graph can be described by
$\mathscr{G}=\{\mathscr{V}, \mathscr{E}\}$. Here, $\mathscr{V}=\{1,2, \ldots, m, m+1, \ldots, n\}$, and it represents the set containing the followers and leaders. $\mathscr{E}=\{(i, j) \in \mathscr{V} \times \mathscr{V}\}$; it embodies the undirected edges between the leader and the followers and neighboring relations among leaders and followers. Denote the set of leaders as $f=\{1,2, \ldots, m\}$ and the set of followers as $l=\{m+1, m+2, \ldots, n\}$. Define the adjacency matrix as $\mathscr{A}=\left[a_{i j}\right] \in \mathbb{R}^{(n) \times(n)}, a_{i j}>0$, if $(j, i) \in \mathscr{E}$, otherwise $a_{i j}=0$.

The Laplacian matrix of the graph is given by $L=\left[L_{i j}\right] \in \mathbb{R}^{(N) \times(N)}$, where $l_{i i}=\sum_{j=1, j \neq i}^{N} a_{i j}$ and $l_{i j}=-a_{i j}$, $i \neq j$.

2.2. Definition and Notations. In this paper, $1_{n}, I_{m}$, and 0 are designed as $n \times 1$ column vector of all ones, the identity matrix with order $m$, and zero matrix, respectively. $\operatorname{diag}\left\{x_{1}, x_{2}, \ldots, x_{n}\right\}$ denotes the block-diagonal matrix constructed by $x_{1}, x_{2}, \ldots, x_{n}$. $\otimes$ is the Kronecker product.

Definition 1. A set $\mathscr{C} \subseteq \mathbb{R}^{(N) \times(N)}$ is convex if, for all $x, y \in \mathscr{C}$ and any $\mu \in[0,1]$, we can have $(1-\mu) x+\mu y \in \mathscr{C}$. Define the minimal convex set encompassing all points $\mathscr{X}=\left\{x_{1}, x_{2}, \ldots, x_{M}\right\}$ as $\operatorname{Co}(\mathscr{X})$. In detail,

$$
\operatorname{Co}(\mathscr{X})=\left\{\sum_{i=1}^{M} \mu_{i} x_{i} \mid x_{i} \in \mathscr{X}, \mu_{i} \in \mathbb{R}, \mu_{i} \geq 0, \sum_{i=1}^{M} \mu_{i}=1\right\} .
$$

\section{Problem Formulation}

In this part, the containment law with the dynamic leader for the heterogeneous MASs is introduced, while each follower agent receives the relative information of its neighbors with time delays $\tau(t)$.

Definition 2. If, for each tracking node, at least one guide node has a directed path to the following node, the directed graph $\mathscr{G}$ has a spanning tree [25].

We consider the discrete-time MASs. Each leader's dynamics is described as follows:

$$
x_{i}(k+1)=x_{i}(k)+T u_{i}(k), \quad i=1, \ldots, m .
$$

The dynamics of the followers are given as follows:

$$
\left\{\begin{array}{l}
x_{i}(k+1)=x_{i}(k)+T v_{i}(k), \quad i=m+1, \ldots, n, \\
v_{i}(k+1)=v_{i}(k)+T u_{i}(k),
\end{array}\right.
$$

where $T$ is the sample time.

The Laplacian matrix $\mathscr{L}$ can be written as

$$
L=\left[\begin{array}{cc}
L_{l l} & L_{f l} \\
0 & L_{f f}
\end{array}\right]
$$

where $L_{l l}$ is the corresponding Laplacian matrix among leaders, $L_{f f}$ is the Laplacian matrix among followers, and $L_{f l}$ represents the connection between leaders and followers. Note that the last $n-m$ rows and the first $m$ columns of $\mathscr{L}$ are equal to zero because the last $n-m$ rows are for the 
followers and the first $m$ columns represent the leaders, who do not receive the information from any other agents.
In order to solve the containment problem for heterogeneous agents, a distributed controller is designed. The control protocol is illustrated as follows:

$$
\begin{cases}u_{i}(k)=\sum_{j=1}^{m} a_{i j}\left[x_{j}(k-\tau(k))-\eta_{j}-\left(x_{i}(k-\tau(k))-\eta_{i}\right)\right], & i=1, \ldots, m, \\ u_{i}(k)=\sum_{j=1}^{n}\left[x_{j}(k-\tau(k))-x_{i}(k-\tau(k))\right]+\gamma^{-1} \sum_{j=1}^{n} a_{i j}\left[v_{j}(k-\tau(k))-v_{i}(k-\tau(k))\right]-\gamma v_{i}(k), & i=m+1, \ldots, n,\end{cases}
$$

where $\gamma=k_{1}^{-1}, k_{1}>0$, is an unknown feedback gain which Letting $y_{i}(t)=x_{i}(t)+\gamma^{-1} v_{i}(t)$, we have awaits for later determination.

$$
\left\{\begin{array}{l}
x_{f}(k+1)=x_{f}(k)+T \gamma\left[y_{f}(k)-x_{f}(k)\right] \\
y_{f}(k+1)=y_{f}(k)-T\left[\gamma^{-1} L_{f f} y_{f}(k-\tau(k))+\gamma^{-1} L_{f l} y_{l}(k-\tau(k))\right], \\
x_{l}(k+1)=x_{l}(k)-T L_{l l} x_{l}(k-\tau(k))+T L_{l l} \eta_{l}(k)
\end{array}\right.
$$

where $x_{f}=\left[x_{m+1}, x_{m+2}, \ldots, x_{n}\right], y_{f}=\left[y_{m+1}, y_{m+2}, \ldots, y_{n}\right]$, $x_{l}=\left[x_{1}, x_{2}, \ldots, x_{m}\right]$, and $y_{l}=\left[y_{1}, y_{2}, \ldots, y_{m}\right] . y_{i}(t), i=m$ $+1, \ldots, n$, is considered as virtual followers corresponding to $x_{i}(t), i=m+1, \ldots, n$.

Noting that the Laplacian matrix of a directed graph is asymmetrical, thus its corresponding eigenvalues are complex. For the convenience, we state the following lemma to provide a rational for further demonstration.

Lemma 1 (see [26]). Suppose that the digraph $\mathscr{G}$ has a directed spanning tree. Then, all the eigenvalues of $L_{f f}$ have positive real parts, each element of $L_{f f}^{-1} l_{f l}$ is nonnegative, and the sum of each row of $L_{f f}^{-1} l_{f l}$ is 1 .

Lemma 2 (see [27]). Given the polynomial with complex coefficients,

$$
F_{n}(s)=s^{n}+\left(\alpha_{1}+i \beta_{1}\right) s^{n-1}+\cdots+\left(\alpha_{n-1}+i \beta_{n-1}\right) s+\alpha_{n}+i \beta_{n} .
$$

The polynomial is stable if and only if $F_{n-1}(s)$ (see equation (11)) is stable:

$$
\begin{aligned}
F_{n-1}(s)= & \alpha_{1} s^{n-1}+\left(\alpha_{1} \alpha_{2}^{(1)}+\beta_{1}^{(1)} \beta_{2}+i \beta_{2}\right) s^{n-1}+\cdots+ \\
& {\left[\alpha_{n-1}+i\left(\alpha_{1} \beta_{n-1}^{(1)}-\beta_{1}^{(1)} \alpha_{n-1}\right)\right] s+\alpha_{1} \alpha_{n}^{(1)}+\beta_{1}^{(1)} \beta_{n}+i \beta_{n}, }
\end{aligned}
$$

where $\beta_{1}^{(1)}=\alpha_{1} \beta_{1}-\beta_{2}, \quad \alpha_{2}^{(1)}=\alpha_{1} \alpha_{2}-\alpha_{3}, \ldots, \beta_{n-1}^{(1)}=\alpha_{1} \beta_{n}$ $-1-\beta_{n}, \alpha_{n}^{(1)}=\alpha_{1} \alpha_{n}$.

Corollary 1. It is not difficult to verify that Lemma 2 works well for a second-order polynomial $s^{2}+p s+q$, whose conditions can be induced as

$$
\left\{\begin{array}{l}
\operatorname{Re}(p)>0 \\
(\operatorname{Re}(p))^{2} \operatorname{Re}(q)+\operatorname{Re}(p) \operatorname{Im}(p) \operatorname{Im}(q)-(\operatorname{Im}(q))^{2}>0 .
\end{array}\right.
$$

\section{Main Results}

The sufficient condition proves that law (6) is competent with the consensus objective of heterogeneous MAS (3) and (5).

Theorem 1. Suppose that the sampling time $T$ satisfies $T<1 / \max _{i=1, \ldots, n} d_{i i}$. Using (6) for (3) and (5), the heterogeneous MASs reach consensus, for any initial condition, if $\mathscr{G}$ contains a directed spanning tree, feedback gain $\gamma^{-1}$ and sample time $T$ satisfy

$$
\left\{\begin{array}{l}
T<\gamma^{-1} \\
4\left|\mu_{i}\right|^{4}\left(T-\gamma^{-1}\right)^{4}-\left[4 \gamma^{-2}\left|\mu_{i}\right|^{4}-12 \operatorname{Re}\left(\mu_{i}\right)\left|\mu_{i}\right|^{2}\right]\left(T-\gamma^{-1}\right)^{2}-9 \operatorname{Im}^{2}\left(\mu_{i}\right)>0
\end{array}\right.
$$

and time delay, 


$$
\tau \in[0, \bar{\tau}), \quad \bar{\tau}=\min _{\mu_{i}, i \in f}\{\tau|| Z \mid \geq 1, \Psi(z, \tau)=0\} .
$$

Proof. Taking the $Z$ transform of $x_{l}$ in equation (2); then, we can obtain

$$
X_{f}(z)=(z-1+T \gamma)^{-1}\left[T \gamma Y(z)+z x_{f}(0)\right] .
$$

Similarly, applying the $Z$ transform to equation (3), we can obtain

$$
\begin{aligned}
& {\left[\left(z^{2}-2 z+1\right) I_{m}+\left(T^{2}-T \gamma^{-1}\right) L_{f f} z^{-\bar{\tau}}+\left(T \gamma^{-1}\right) L_{f f} z^{-\bar{\tau}+1}\right] Y_{f}(z) } \\
= & z(z-1+T \gamma) y(0)-T \gamma x_{f}(0)-\left(T \gamma^{-1}\right)(z-1+T \gamma) L_{f l} \frac{z^{-\bar{\tau}+1}}{z-1 x_{l}}
\end{aligned}
$$

where $X_{f}(z)$ and $Y_{f}(z)$ are the $Z$ transforms of $x_{f}(k)$ and $y(k)$, respectively.

Firstly, we show that the convergence of the state of real followers will reach to the state of their own virtual followers. We have $T-\gamma^{-1}<0$. The condition and final value theorem guarantee the response of (13), i.e., $x_{f}(k)$, where the initial condition $x_{f}(0)$ will be 0 at $t \longrightarrow \infty$. Besides, (14) indicates that the poles of $Y(z)$ are the zeros of the transfer matrix:

$$
\left(z^{2}-2 z+1\right) I_{m}+\left(T^{2}-\frac{T}{\gamma}\right) L_{f f} z^{-\bar{\tau}}+\frac{T}{\gamma} L_{f f} z^{-\bar{\tau}+1}
$$

It can be completed by calculating characteristic equation in the following:

$$
\prod_{i=1}^{m}\left(z^{2}-2 z+1+\left(T^{2}-\frac{T}{\gamma}\right) \mu_{i} z^{-\bar{\tau}}+\frac{T}{\gamma} z^{-\bar{\tau}+1}\right)=0 .
$$

Case 1: $\tau=0$.

Assume that there is no time delay; equation (15) will be reduced to

$$
z^{2}+\left(\frac{T}{\gamma} \mu_{i}-2\right) z+1+\left(T^{2}-\frac{T}{\gamma}\right) \mu_{i}=0
$$

All the roots of the polynomial,

$$
z^{2}+\left(\gamma^{-1} T \mu_{i}-2\right) z+1+\left(T^{2}-\gamma^{-1} T\right) \mu_{i}=0,
$$

site in the unit circle if and only if the sample time $T$ satisfies the following inequality:

$$
\left\{\begin{array}{l}
T<\gamma^{-1}, \\
4\left|\mu_{i}\right|^{4}\left(T-\gamma^{-1}\right)^{4}-\left[4 \gamma^{-2}\left|\mu_{i}\right|^{4}-12 \operatorname{Re}\left(\mu_{i}\right)\left|\mu_{i}\right|^{2}\right]\left(T-\gamma^{-1}\right)^{2}-9 \operatorname{Im}^{2}\left(\mu_{i}\right)>0,
\end{array}\right.
$$

The proof is as follows. In order to keep the roundness of the polynomial equation, the value out of the unit circle is mapped to its right, and we substitute $z$ in equation (17) by adopting a Mobius transformation, i.e., $z \longrightarrow s+1 / s-1$; then, multiplying $(s-1)^{2}$ to the two sides of equation (17), subsequently, equation (17) becomes

$\left(T^{2} \mu_{i}\right) s^{2}+2 \mu_{i}\left(\gamma^{-1} T-T^{2}\right) s+\left[\left(T^{2}-2 \gamma^{-1} T\right) \mu_{i}+3\right]=0$.

It can be derived that equation (19) follows the conditions in equation (18). Then, use Lemma 1 , if and only if equation (19) is stable, which is equivalent to equation (17) which has roots inside the unit circle.

All the roots of characteristic polynomial are inside the unit circle. Hence, this system is asymptotically stable based on the final value theorem.

Case 2: $\tau \neq 0$.

When $T<\gamma^{-1}$, we can have [28]

$$
\Psi(z, \tau)=\prod_{i=1}^{m}\left(z^{2}-2 z+1+\left(T^{2}-\frac{T}{\gamma}\right) \mu_{i} z^{-\bar{\tau}}+\frac{T}{\gamma} \mu_{i} z^{-\bar{\tau}+1}\right)=0 .
$$

It has no root locating inside the unit circle for $\forall \tau \in \mathbb{Z}$.

It can be easily found that the roots of (22) never stay over the unit circle for $\forall \tau \in \mathbb{Z}$. Therefore, one can guarantee that the system never makes the oscillation behavior cross the convex hull spanned by the leaders' states. Hence, the maximum time delay can be reckoned by $\tau$. Therefore, when the delay satisfies $\tau$, (12) and (22) are stable.

Thus, it is sufficient to prove the convergence of virtual follower to the convex hull crossed by the leader as $t \longrightarrow \infty$; it further demonstrates that the real followers are able to converge to the convex hull which is spanned by the leaders.

Supposing that

$$
\begin{aligned}
\xi(k) & =\left[x(k), x(k-1), \ldots, x\left(k-\left\lceil\frac{\bar{\tau}}{T}\right\rceil\right)\right]^{T}, \\
x(k-j) & =\left[x_{1}(k-j), \ldots, x_{m}(k-j), y_{m+1}(k-j), \ldots, y_{n}(k-j)\right]^{T},
\end{aligned}
$$

the equation of the system can be summarized as follows:

$$
\xi(k+1)=\Phi \xi(k)+Y,
$$

where 


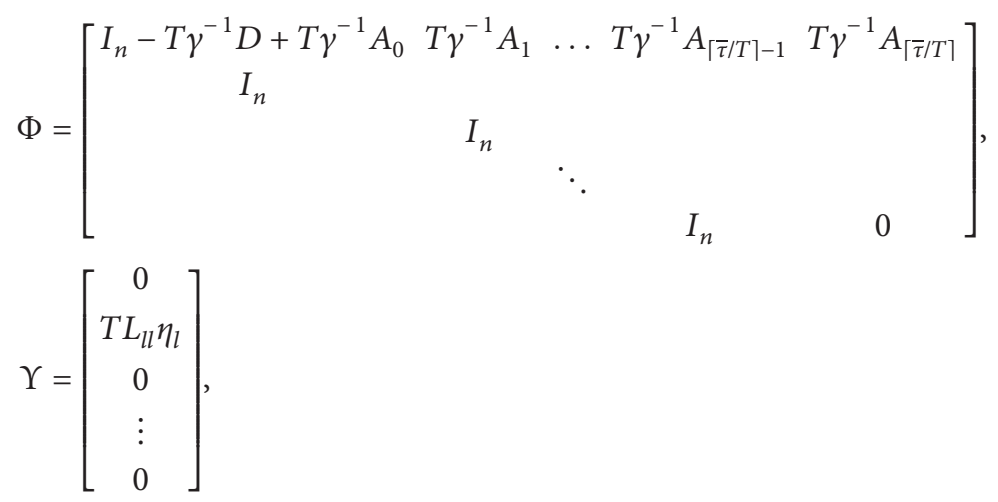

where the Laplacian matrix $L=D-\sum_{k=0}^{[\tau / T]} A_{k}$.

Meanwhile, from the definitions of the leaders and the followers, it is easy to attain the following partitions:

$$
\begin{aligned}
D & =\left[\begin{array}{ll}
D_{f} & \\
& D_{l}
\end{array}\right], \\
A_{k} & =\left[\begin{array}{cc}
A_{f f k} & A_{f l k} \\
\mathbf{0} & A_{l l k}
\end{array}\right] .
\end{aligned}
$$

Denote $\xi_{f}(k)=\left[y_{f}^{T}(k), y_{f}^{T}(k-1), \ldots, y_{f}^{T}(k-\lceil\bar{\tau} / T\rceil)\right]^{T}$ and $\xi_{l}(k)=\left[x_{l}^{T}(k), x_{l}^{T}(k-1), \ldots, x_{l}^{T}(k-\lceil\bar{\tau} / T\rceil)\right]^{T}$. Afterwards, the dynamics of the leaders can be transformed to

$$
\xi_{l}(k+1)=\Phi_{l l} \xi_{l}(k)+Y_{l},
$$

(24) where

$$
\begin{aligned}
\Phi_{l l} & =\left[\begin{array}{ccccc}
I_{m}-T D_{l}+T A_{l l 0} & T A_{l l 1} & \ldots & T A_{l l([\bar{\tau} / T]-1)} & T A_{l l[\bar{\tau} / T]} \\
I_{m} & I_{m} & & & \\
& & \ddots & & \\
& & & I_{m} & 0
\end{array}\right], \\
\Upsilon_{l} & =\left[\begin{array}{c}
T L_{l l} \eta_{l} \\
0 \\
0 \\
\vdots \\
0
\end{array}\right] .
\end{aligned}
$$

Likewise, the dynamics of the followers become

$$
\xi_{f}(k+1)=\Phi_{f f} \xi_{f}(k)+\Phi_{f l} \xi_{l}(k),
$$

where

$$
\begin{aligned}
& \Phi_{f f}=\left[\begin{array}{cccc}
I_{n-m}-T D_{f}+T A_{f f 0} & T A_{f f 1} & T A_{f f([\bar{\tau} / T]-1)} & T A_{f f[\bar{\tau} / T]} \\
I_{n-m} & & \\
I_{n-m} & & & \\
\ddots & 0 &
\end{array}\right] \text {, } \\
& \Phi_{f f}=\left[\begin{array}{ccccc}
T A_{f l 0} & T A_{f l 1} & \ldots & T A_{f l([\bar{\tau} / T]-1)} & T A_{f l[\bar{\tau} / T\rceil} \\
0 & 0 & \ldots & 0 & 0 \\
0 & 0 & \ldots & 0 & 0 \\
\vdots & \vdots & \ddots & \vdots & \vdots \\
0 & 0 & \ldots & 0 & 0
\end{array}\right] .
\end{aligned}
$$




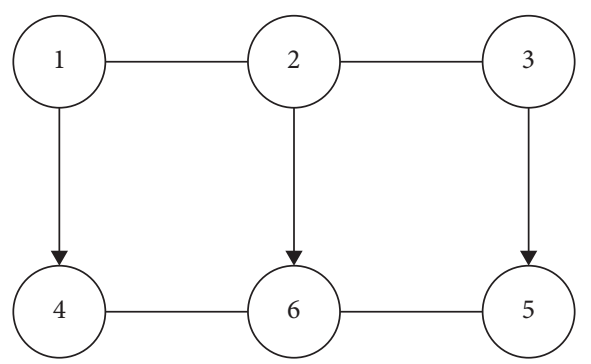

(a)

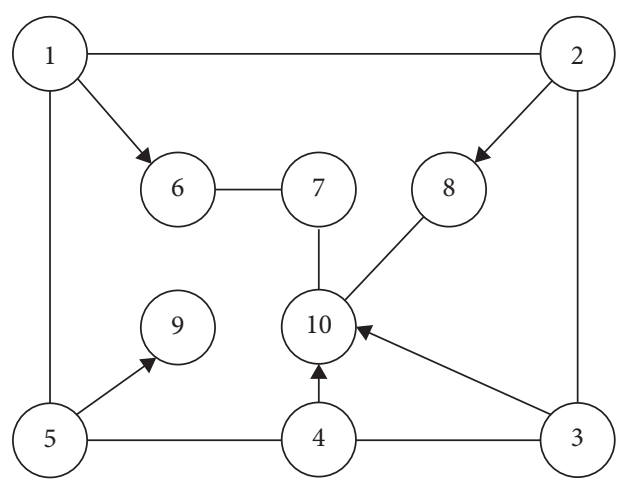

(b)

Figure 1: The communication topology of two examples. (a) Example 1. (b) Example 2.

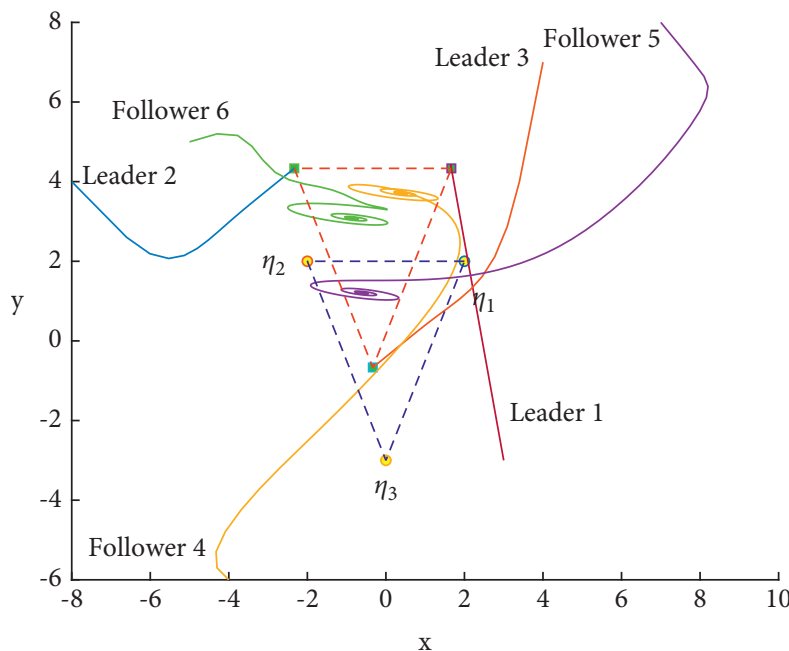

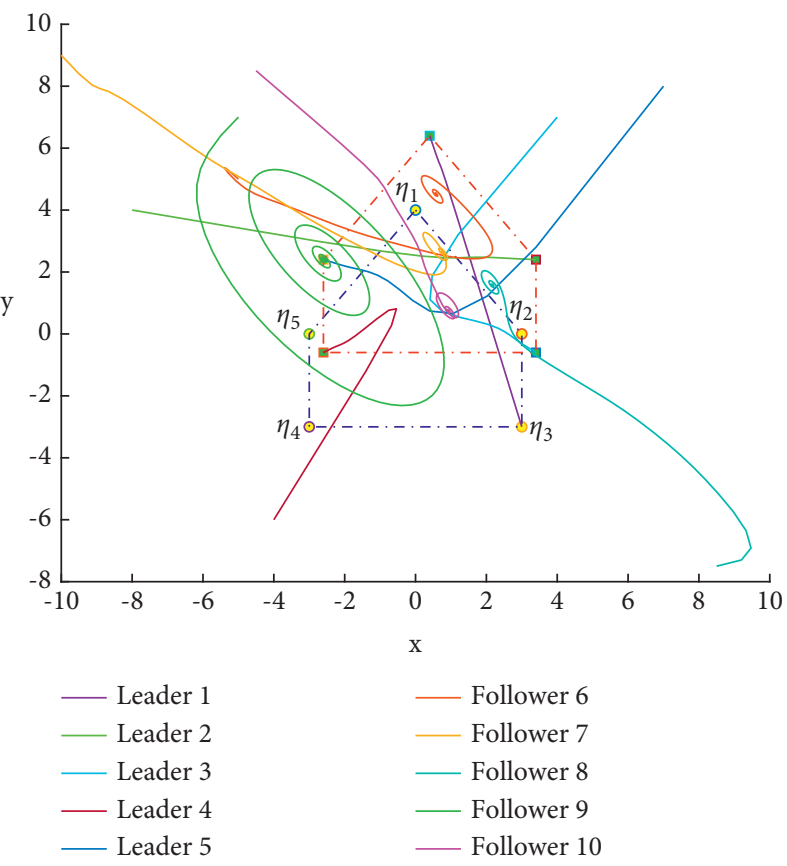

(b)

Figure 2: State trajectories of all agents in the phase plane. (a) Example 1. (b) Example 2.

It is worth mentioning that $T<1 / \max _{i=1, \ldots, n} d_{i i}$; therefore, $\rho\left(\Phi_{f f}\right) \leq 1$. Assume that 1 is an eigenvalue of $\Phi_{f f}$; it can be concluded that there exists an eigenvector,

$$
\alpha=\left[\alpha_{0}^{T}, \alpha_{1}^{T}, \ldots, \alpha_{\lceil\bar{\tau} / T\rceil}^{T}\right]^{T} \neq 0
$$

such that $\Phi_{f f} \alpha=1 \cdot \alpha$, i.e.,

$$
\begin{aligned}
& \left(I_{n-m}-T D_{f}+T A_{f f 0}\right) \alpha_{0}+T A_{f f 1} \alpha_{1}+\cdots+T A_{f f[\bar{\tau} / T]} \alpha_{[\bar{\tau} / T]}=\alpha_{0}, \\
& \alpha_{0}=\alpha_{1}, \\
& \quad \vdots \\
& \alpha_{[\bar{\tau} / T]-1}=\alpha_{[\bar{\tau} / T]} .
\end{aligned}
$$




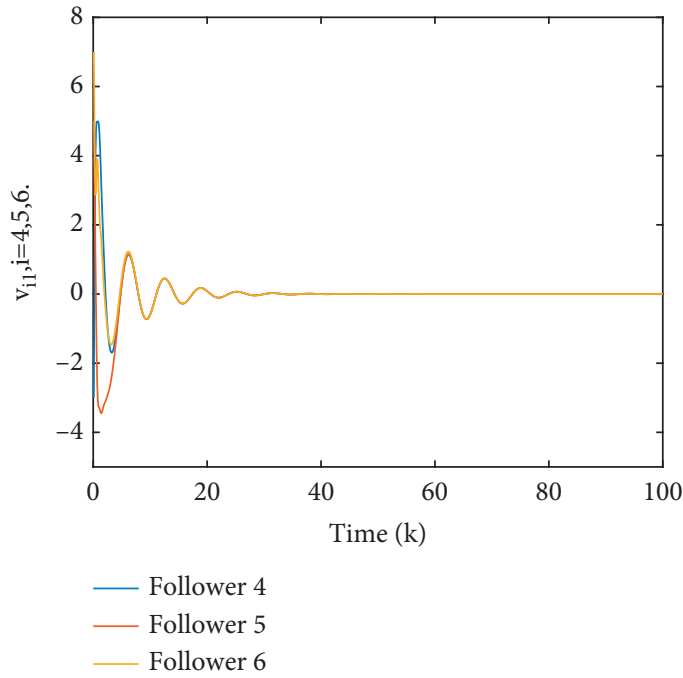

(a)

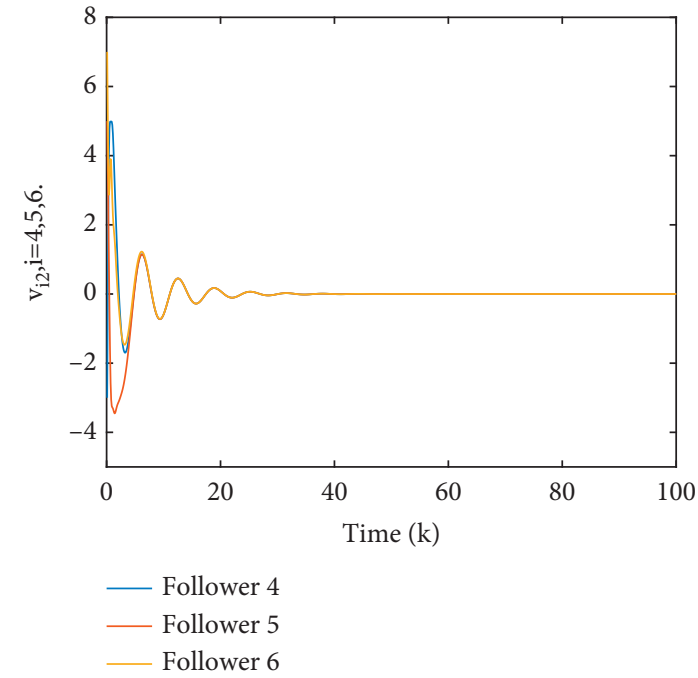

(b)

Figure 3: Velocity trajectories of all followers in Example 1. (a) Case 1. (b) Case 2.

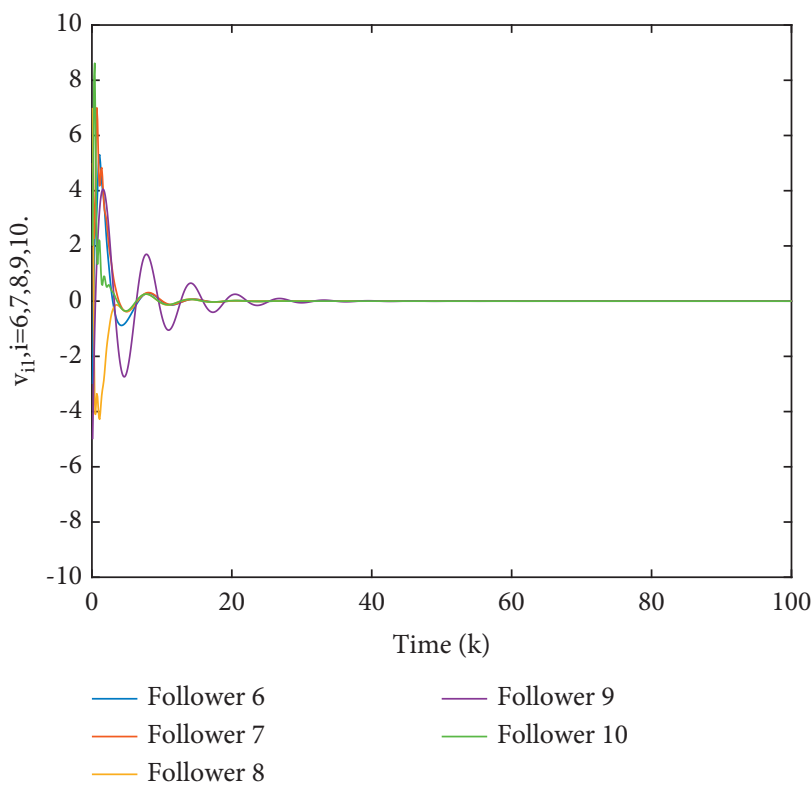

(a)

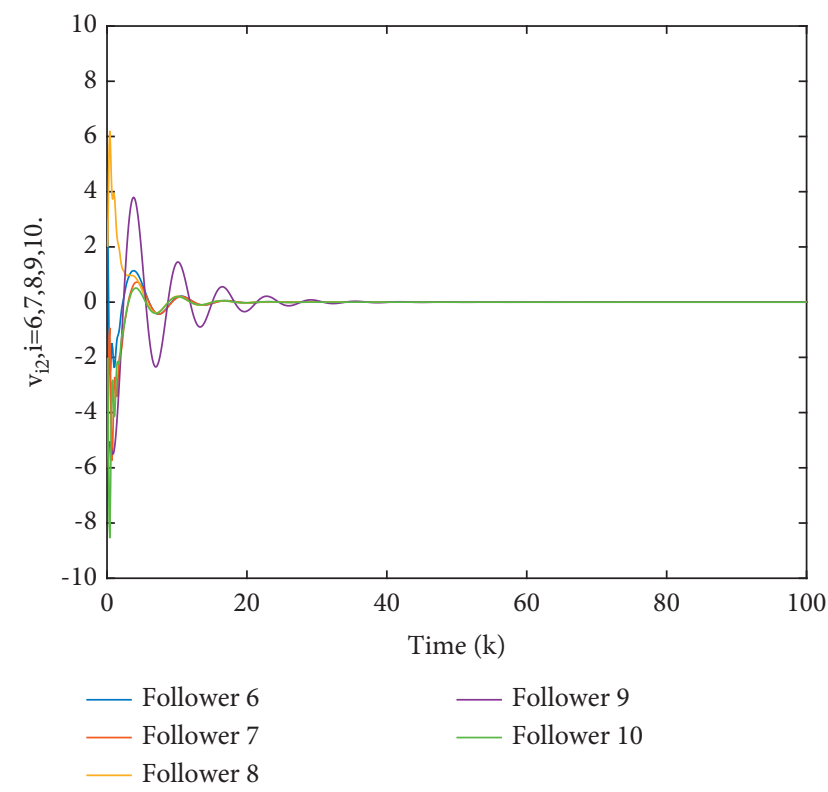

(b)

Figure 4: Velocity trajectories of all followers in Example 2. (a) Case 1. (b) Case 2. 
From equation (30a), we can directly obtain that $\left(I_{n-m}-T L_{f f}\right) \alpha_{0}=\alpha_{0}$, that is, $T L_{f f} \alpha_{0}=0$. Thereon, it follows that $\alpha=0$. It arrives to a contradiction, where $\alpha \neq 0$ is assumed in equation (29). Hence, every eigenvalue of $\Phi_{f f}$ is less than 1.

Once the leaders achieved the desired information, the states of virtual followers would become

$$
\begin{aligned}
\lim _{k \longrightarrow \infty} \xi_{f}(k+1) & =\lim _{k \longrightarrow \infty}\left[\Phi_{f f}^{k+1} \xi_{f}(0)+\left(\Phi_{f f}^{k} \Phi_{f l}+\Phi_{f f}^{k-1} \Phi_{f l}+\cdots+\Phi_{f f} \Phi_{f l}+\Phi_{f l}\right) \xi_{l}\right] \\
& =\lim _{k \longrightarrow \infty}\left[\Phi_{f f}^{k+1} \xi_{f}(0)+\sum_{i=0}^{k} \Phi_{f f}^{i} \Phi_{f l} \xi_{l}\right] \\
& =\left(I_{(n-m) \times([\bar{\tau} / T]+1)}-\Phi_{f f}\right)^{-1} \Phi_{f l} \xi_{l} \\
& =\Gamma \xi_{l},
\end{aligned}
$$

where $\Gamma=1_{[\bar{\tau} / T]+1} \otimes\left[L_{f f}^{-1} A_{f l 0}, L_{f f}^{-1} A_{f l 1}, \ldots, L_{f f}^{-1} A_{f l[\bar{\tau} / T]}\right] \xi_{l}$. by

Finally, the positions of the virtual followers can be given

$$
\begin{aligned}
y_{f} & =\left[L_{f f}^{-1} A_{f l 0}, L_{f f}^{-1} A_{f l 1}, \ldots, L_{f f}^{-1} A_{f l\lceil\bar{\tau} / T]}\right] \xi_{l} \\
& =L_{f f}^{-1} A_{f l} x_{l} .
\end{aligned}
$$

It is not difficult to verify that $L_{f f}^{-1} A_{f l 1} 1_{m}=1_{n-m}$. Besides, all the entries of $L_{f f}^{-1} A_{f l 1}$ are nonnegative. Considering the definition of convex hull, the result reveals that all of the followers converge to the convex hull spanned by the leaders' final states.

\section{Simulation Results}

5.1. Example 1. First of all, considering a containment problem of discrete-time multiagent systems (DTMASs), its communication topology graph is shown in Figure 1(a), where $l=\{1,2,3\}$ and $f=\{4,5,6\}$. It is evident that each follower has at least one directed path to the leaders. To avoid stochastic factors, two sets of parameters are tested on this example.

In the first case of validation, take

$\gamma=0.5, T=0.1, \eta_{1}=(2,2)^{T}, \eta_{2}=(-2,2)^{T}, \eta_{3}=\quad(0$, $-3)^{T}, \tau=0.1 / T, x_{1}(0)=(3,-3)^{T}, x_{2}(0)=(-8,4)^{T}, x_{3}(0)=$ $(4,7)^{T}, x_{4}(0)=(-4,-6)^{T}, x_{5}(0)=(7,8)^{T}$, and $x_{6}(0)=$ $(-5,5)^{T}$.

In the second case of validation, take $\gamma=0.5, T=$ $0.1, \eta_{1}=(2,2)^{T}, \eta_{2}=(-2,2)^{T}, \eta_{3}=(0,-3)^{T}, \quad \tau=0.1 / T$, $x_{1}(0)=(3,-3)^{T}, x_{2}(0)=(-8,4)^{T}, x_{3}(0)=(4,7)^{T}, x_{4}(0)=$ $(-3,3)^{T}, x_{5}(0)=(5,6)^{T}$, and $x_{6}(0)=(7,2)^{T}$.

No matter in which case, the state trajectories of all agents $(t \rightarrow \infty)$ in the phase plane are presented in Figure 2(a), and the velocity trajectories of all followers in these two cases over time are depicted in Figures 3(a) and 3(b), respectively. It can be seen from Figures 2(a) and Figure 3 that the containment control of the systems can be achieved without the influence of initial positions.

5.2. Example 2. Now, take a more complex system into consideration, similar to example 1 , but more vertices are added. Its communication topology graph is shown in
Figure $1(\mathrm{~b})$, where $l=\{1,2,3,4,5\}$ and $f=\{6,7,8,9,10\}$. It is noteworthy that each follower harbors at least one directed path to the leaders. Likewise, two cases are conducted for the confirmation.

In the first case, the parameters are set to be

$T=0.1, \gamma=0.5, \tau=0.1 / T, \eta_{1}=(0,4)^{T}, \eta_{2}=(3,0)^{T}, \eta_{3}=$ $(3,-3)^{T}, \eta_{4}=(-3,-3)^{T}, \eta_{5}=(-3,0)^{T}, x_{1}(0)=(3,-3)^{T}$, $x_{2}(0)=(-8,4)^{T}, x_{3}(0)=(4,7)^{T}, \quad x_{4}(0)=(-4,-6)^{T}, \quad x_{5}$ $(0)=(7,8)^{T}, x_{6}(0)=(-5,5)^{T}, x_{7}(0)=(-10,9)^{T}, x_{8}(0)=$ $(8.5,-7.5)^{T}, x_{9}(0)=(-5,7)^{T}$, and $x_{10}(0)=(-4.5,8.5)^{T}$.

In the second case, the parameters are set to be $T=0.1, \gamma=0.5, \tau=0.1 / T, \eta_{1}=(0,4)^{T}, \eta_{2}=(3,0)^{T}$, $\eta_{3}=(3,-3)^{T}, \quad \eta_{4}=(-3,-3)^{T}, \eta_{5}=(-3,0)^{T}, \quad x_{1}(0)=(3$, $-3)^{T}, x_{2}(0)=(-8,4)^{T}, x_{3}(0)=(4,7)^{T}, x_{4}(0)=(-4,-6)^{T}$, $x_{5}(0)=(7,8)^{T}, x_{6}(0)=(-3,3)^{T}, x_{7}(0)=(5,-6)^{T}, x_{8}(0)=$ $(7,2)^{T}, x_{9}(0)=(-5,-6)^{T}$, and $x_{10}(0)=(2,-2)^{T}$.

In both cases, when $t \rightarrow \infty$, the state trajectories of all agents in the phase plane are illustrated in Figure 2(b), and the velocity trajectories of all followers in these two cases over time are depicted in Figures 4(a) and 4(b), respectively. From Figures 2(b) and 4, it can be seen that the containment control of the systems can be achieved with the number of agents increasing. The results unveil that the control protocol is efficient and robust for the containment problem.

\section{Conclusion}

This paper studies the containment control of heterogeneous discrete-time multiagent systems with time delay. In this manuscript,

(1) The double-integrator followers and single-integrator leaders are investigated.

(2) The containment control problem is of two layers. For the leaders, the information among multiple leaders is interactive and the leaders converge to the expected values. As for the followers, the states converge into the convex hull shaped by the final states of the leaders. 
(3) The sufficient conditions of the sampling time, feedback gain, and delay to ensure the consensus control are given by using a reduced-order method.

(4) The maximum allowed time delay is determined by using the Z-transform.

Finally, the validity of our results is guaranteed by the simulation. In the future, our attention will be focused on the problems such as fractional-order dynamics with nonsymmetric time delays.

\section{Data Availability}

The data used to support the findings of the study are available within the article.

\section{Conflicts of Interest}

The authors declare that there are no conflicts of interest regarding the publication of this article.

\section{Acknowledgments}

This work was supported by the National Natural Science Foundation of China, under Grant 61772063, and Beijing Municipal Natural Science Foundation, under Grant Z180005.

\section{References}

[1] T. Vicsek, A. Czirók, E. Ben-Jacob, I. Cohen, and O. Shochet, "Novel type of phase transition in a system of self-driven particles," Physical Review Letters, vol. 75, no. 6, pp. 1226-1229, 1995.

[2] J. Ali, J. Lin, and A. Stephen Morse, "Coordination of groups of mobile autonomous agents using nearest neighbor rules," IEEE Transactions on Automatic Control, vol. 48, no. 6, pp. 988-1001, 2003.

[3] R. Olfati-Saber and R. M. Murray, "Consensus problems in networks of agents with switching topology and time-delays," IEEE Transactions on Automatic Control, vol. 49, no. 9, pp. 1520-1533, 2004.

[4] Y. Zhang and Q. Han, "Network-based synchronization of delayed neural networks," IEEE Transactions on Circuits and Systems I: Regular Papers, vol. 60, no. 3, pp. 676-689, 2013.

[5] Z. Qu, J. Wang, A. Richard, and Hull, "Cooperative control of dynamical systems with application to autonomous vehicles," IEEE Transactions on Automatic Control, vol. 53, no. 4, pp. 894-911, 2008.

[6] X. Liu, P. Guan, and C. W. Chan, "Nonlinear multivariable power plant coordinate control by constrained predictive scheme," IEEE Transactions on Control Systems Technology, vol. 18, no. 5, pp. 1116-1125, 2009.

[7] F.-Y. Wang, Y.-H. Ni, Z.-X. Liu, and Z.-Q. Chen, "Fully distributed containment control for second-order multi-agent systems with communication delay," ISA Transactions, vol. 99, pp. 123-129, 2020.

[8] W. Yu, G. Chen, and M. Cao, "Some necessary and sufficient conditions for second-order consensus in multi-agent dynamical systems," Automatica, vol. 46, no. 6, pp. 1089-1095, 2010.

[9] J. Qin, H. Gao, and W. X. Zheng, "Second-order consensus for multi-agent systems with switching topology and communication delay," Systems \& Control Letters, vol. 60, no. 6, pp. 390-397, 2011.

[10] X. Yi, R. Guo, and Y. Qi, "Stabilization of chaotic systems with both uncertainty and disturbance by the ude-based control method," IEEE Access, vol. 8, pp. 62471-62477, 2020.

[11] L. Liu, B. Li, and R. Guo, "Consensus control for networked manipulators with switched parameters and topologies," IEEE Access, vol. 9, pp. 9209-9217, 2021.

[12] T. Hou, Y. Liu, and F. Deng, "Stability for discrete-time uncertain systems with infinite markov jump and time-delay," Sciece China Information Sciences, vol. 64, no. 5, 2021.

[13] J. Ni, L. Liu, C. Liu, and J. Liu, "Fixed-time leader-following consensus for second-order multiagent systems with input delay," IEEE Transactions on Industrial Electronics, vol. 64, no. 11, pp. 8635-8646, 2017.

[14] G. Wen, W. Yu, Z. Li, X. Yu, and J. Cao, "Neuro-adaptive consensus tracking of multiagent systems with a high-dimensional leader," IEEE Transactions on Cybernetics, vol. 47, no. 7, pp. 1730-1742, 2017.

[15] G. Wen, H. Wang, X. Yu, and W. Yu, "Bipartite tracking consensus of linear multi-agent systems with a dynamic leader," IEEE Transactions on Circuits and Systems II: Express Briefs, vol. 65, no. 9, pp. 1204-1208, 2018.

[16] G. Wen, Y. Zhao, Z. Duan, W. Yu, and G. Chen, "Containment of higher-order multi-leader multi-agent systems: a dynamic output approach," IEEE Transactions on Automatic Control, vol. 61, no. 4, pp. 1135-1140, 2016.

[17] J. Li, W. Ren, and S. Xu, "Distributed containment control with multiple dynamic leaders for double-integrator dynamics using only position measurements," IEEE Transactions on Automatic Control, vol. 57, no. 6, pp. 1553-1559, 2012.

[18] X. Wang, S. Li, and P. Shi, "Distributed finite-time containment control for double-integrator multiagent systems," IEEE Transactions on Cybernetics, vol. 44, no. 9, pp. 1518$1528,2014$.

[19] H. Liu and G. Xie, "Sampled-data based containment control of continuous-time multi-agent systems with switching topology and time-delays," Neurocomputing, vol. 286, pp. 1930, 2018.

[20] L. Shi, J. Shao, M. Cao, and H. Xia, “Asynchronous group consensus for discrete-time heterogeneous multi-agent systems under dynamically changing interaction topologies," Information Sciences, vol. 463-464, pp. 282-293, 2018.

[21] Y. Zheng and L. Wang, "Containment control of heterogeneous multi-agent systems," International Journal of Control, vol. 87, no. 1, pp. 1-8, 2014.

[22] L. Shi, J. Shao, M. Cao, and H. Xia, "Distributed containment of heterogeneous multi-agent systems with switching topologies," Neurocomputing, vol. 312, pp. 41-48, 2018.

[23] X. Quan, P. Lin, W. Ren, C. Yang, and W. Gui, "Containment control for discrete-time multiagent systems with communication delays and switching topologies," IEEE Transactions on Cybernetics, vol. 49, no. 10, pp. 3827-3830, 2019.

[24] X. Dong, Q. Li, R. Zhang, and Y. Zhong, "Formation-containment analysis and design for high-order linear time-invariant swarm systems with time delays," in Proceedings of the 2015 34th Chinese Control Conference (CCC), pp. 6847-6853, Hangzhou, China, July 2015.

[25] Y. Cao and W. Ren, "Containment control with multiple stationary or dynamic leaders under a directed interaction graph," in Proceedings of the 48h IEEE Conference on Decision and Control (CDC) Held Jointly with 2009 28th Chinese 
Control Conference, pp. 3014-3019, Shanghai, China, December 2009.

[26] H. Liu, G. Xie, and L. Wang, "Necessary and sufficient conditions for containment control of networked multi-agent systems," Automatica, vol. 48, no. 7, pp. 1415-1422, 2012.

[27] X. Xie, "Stable polynomials with complex coefficients," in Proceedings of the 1985 24th IEEE Conference on Decision and Control, vol. 324-325, Fort Lauderdale, FL, USA, December 1985.

[28] M. Asgari and H. Atrianfar, "Necessary and sufficient conditions for containment control of heterogeneous linear multi-agent systems with fixed time delay," IET Control Theory \& Applications, vol. 13, no. 13, pp. 2065-2074, 2019. 